\title{
Radiological Intervention and the Budd-Chiari Syndrome
} JF Griffith

SP Olliff FRCR(UK)

Department of Radiology, Queen Elizabeth Hospital, Birmingham, UK, B152TH

\section{Introduction}

Budd-Chiari syndrome (BCS) is characterised by obstruction of hepatic venous outflow and comprises a range of different clinical presentations, venous abnormalities, therapeutic options and clinical outcomes. Venous obstruction may be in the small centrilobular hepatic veins, in the main hepatic veins or in the distal IVC.

\section{Clinical presentation}

The presenting symptoms depend on the dominantsite of obstruction. If this is the hepatic veins, patients usually present acutely with progressive hepatic engorgement, hepatic impairment and portal hypertension cumulating in hepatic encephalopathy. ${ }^{1,2}$ Conversely, if the dominant site of obstruction is the IVC, patients present with a chronic history of lower limb swelling dating backmany years. ${ }^{1,2}$

\section{Aetiology}

Aetiology also varies according to the principal site of obstruction. In the West, most patients with BCS have an obstruction to their hepatic veins (either centrilobular or main). In about one-third, the cause is unknown, ${ }^{3}$ while most of the remainder have an underlying myeloproliferative disorder. ${ }^{4}$ In theEast, the dominant site of obstruction is usually the IVC and only a minority have an underlying myeloproliferative disorder. ${ }^{5-10}$ IVC obstruction may be a sequel of a congenital venous web anomaly frequently complicated by thrombosis. ${ }^{5-10}$ It is classified as membranous (i.e."caval web"), if it extends over 1-2mm, or segmental if it extends over a few centimetres. IVCobstruction in BCS may also be due to caudate lobe hypertrophy, direct tumour invasion or extrinsic compression by hepatic tumour.

\section{Diagnosis}

The diagnosis ultimately rests on the demonstration of hepatic vein obstruction. We now rarely perform hepatic scintigraphy ${ }^{11}$ preferring to rely on ultrasound (Figures la,b). ${ }^{12-14}$ Ultrasound evaluates hepatic and portal vein patency, liver morphology

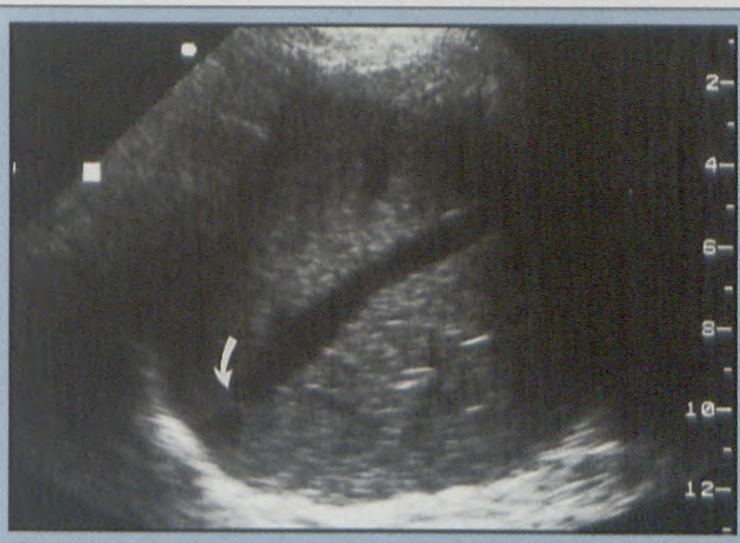

Figure 1a: Longitudinal liver ultrasound. Para-ostial stenosis (arrowed) of distended right hepatic vein with (b) monophasic flow on duplex study.

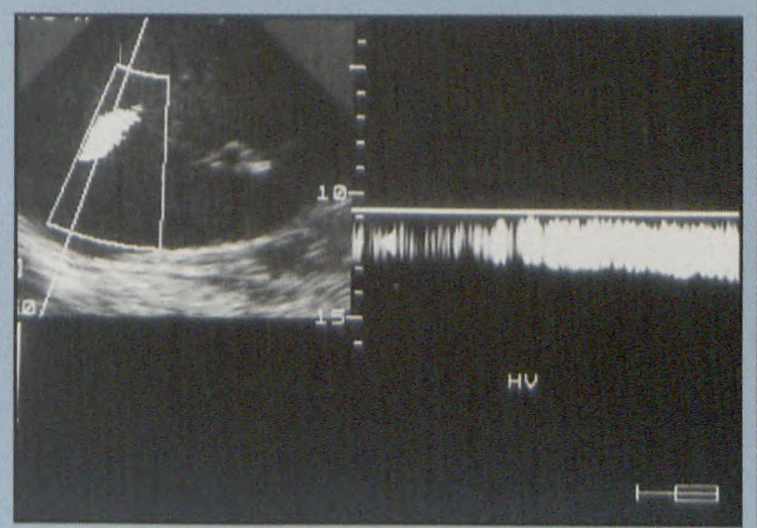

Figure 1b: Monophasic flow on duplex study. 
(eg.caudatelobehypertrophy) andfeatures of portal hypertension. The hepatic veins may be engorged, irregular, filled with thrombus or absent. Triphasic flow may be dampened (Figure lb) or reversed. Collaterals (intrahepatic, subcapsular) with a characteristic curved or "hockey stick" appearance may be visible. The IVC may be narrowed, displaced by caudate hypertrophy or contain intraluminal thrombus. ${ }^{12-14}$

Venography remains the gold standard diagnostic tool (Figures 2a, 5a and 6a,b).

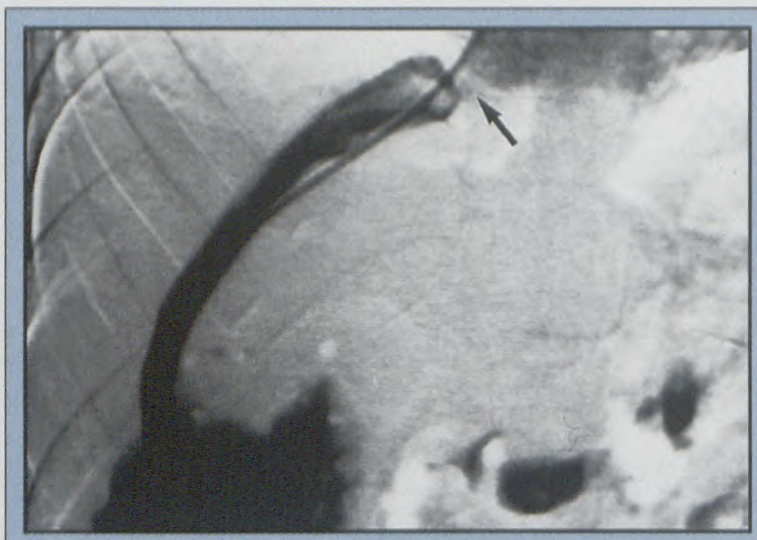

Figure 2a: Right hepatic venogram in the same patient as Figure 1.(a) There is a short segment stenosis near the hepatic vein ostium (arrow).

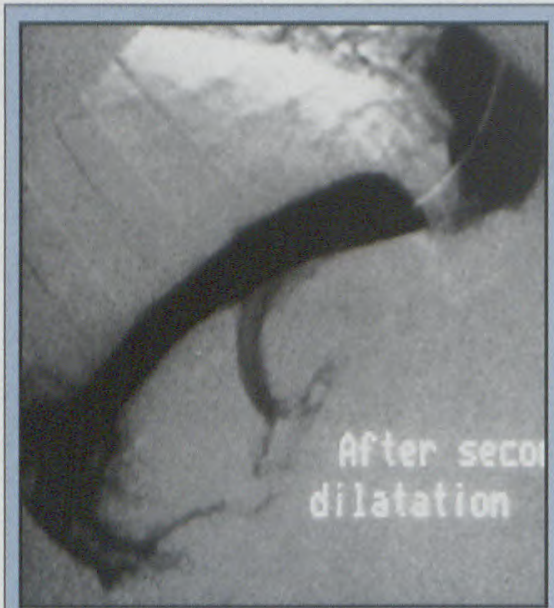

Figure 2.(b) Following dilatation, the stenosis is still clearly visible though flow (and pressure gradient) improved. The patient also clinically improved.
Inferior venography is initially performed to assess intrinsic IVC obstruction or significant extrinsic compression which may be present in sixty five per cent. ${ }^{3}$ The exact length, location and direction of obstruction can be assessed by simultaneous injections of catheter placed on either side of the obstruction. ${ }^{15}$ Pressure measurements are obtained from the infrahepatic, intrahepatic and suprahepatic IVC as an adjunct to surgical planning.

A transjugular approach allows easiest access to the hepatic veins. The walls of the IVC should be genty probed with a suitably shaped catheter to locate the hepatic venous ostia. Flush inferior vena cavography alone cannot be relied upon to opacify severely stenosed venous ostia. Once cannulated, free and wedged venous measurements are obtained to give an assessment of portal hypertension, the hepatic wedged venous pressure correlating roughly with the degree of portal hypertension. Hepatic venography allows assessment of venous calibre, stenoses and thrombosis of either thelarge or small veins (the latter evidenced by a "spider's web" appearance which reflects partially recanalised and collaterised third and fourth order venules). A liver biopsy may be taken at the same time. This allows assessment of hepatic fibrosis and differentiates BCS-type changes ("perisinusoidal congestion") from hepatic venocclusive-type change where the terminal hepatic venules are obliterated.

\section{Venographic abnormalities at presentation}

In patients with dominant hepatic venous obstruction, the degree of limitation of hepatic venous flow at presentation is striking. Themost venographic patterns evident at presentation are (i) a "spider's web" appearance alone without obstruction to the main hepatic veins, (ii) complete occlusion of all three veins or (iii) an occlusion of two out of three veins with a severe stenosis of the remaining vein. A critical level of hepatic vein occlusion must be reached before symptoms develop. This is supported by a prevalence of asymptomatic hepatic venous obstruction present in postmortems of $0.183 \% .{ }^{16}$ Intrahepatic collaterals will usually be established at presentation. Similarly, with dominant IVC obstructions, a short ("membranous") or long segment ("segmental") stenosis/occlusion with established azygos and hemiazygos collaterals is typical, accompanied by varying degrees of obstruction to the main hepatic veins.

\section{Treatment options}

Thismay besupportivemedical treatment, surgical or radiological.

\section{Medical management}

Medical management involves treating underlying myeloproliferative disorders, anticoagulation of hypercoagulablepatients, control of ascites and lactulose/dietary restriction to treat hepatic encephalopathy.

\section{Surgical treatment}

Surgical management consists of peritoneovenous shunts, dorsocranial liver resection with venous reconstruction, portosystemic shunting, direct venous repair and orthotopic liver transplantation. ${ }^{3}$ Mesocaval shunts (which involve interposition of an autologous or dacron graft between the IVC and the superior mesenteric vein) are unsuitable for patients with high caval pressures. Mesoatrial shunts (preferred if the IVC pressure is high) involve interposition of a long narrow polyethylene prosthesis between the superior mesenteric vein and the 
right atrium over the liver surface. These grafts are prone to occlusion due to low flow states and graft thrombogenicity. ${ }^{3}$ Nevertheless, the 5-year survival for patients undergoing mesocaval or mesoatrial shunts is $60-75 \%{ }^{3}$ Hepatic transplantation is reserved for those patients with advanced hepatic fibrosis.

\section{Radiological intervention}

Since percutaneous angioplasty of an IVC web was described in $1974,{ }^{19}$ there have been many reports heralding radiological intervention as a successful treatment ${ }^{1,3,6,18-39}$ for BCS. The diversity of radiological options includes standard angiographic dilatation; , 1,2, $, 15,17,20$ 22,24,26,28,30,31,38IVC recanalisation utilising a straight transeptal needle, ${ }^{6}$ endomyocardial biopsy device, ${ }^{18}$ stiff guide wire ${ }^{24}$ or laser; ${ }^{27}$ hepatic vein recanalisations utilising a transhepatic approach;, ${ }^{1,29,37}$ percutaneous stent insertion, ${ }^{1,19,32,33,37}$ thrombolysis, ${ }^{23,25}$ transluminal intrahepatic portosystemic shunting (TIPS) ${ }^{34-37}$ and portosystemic shunt dilatation. ${ }^{38}$

\section{Patient suitability for radiological intervention}

We reviewed 47 patients admitted over an eight year period with BCS. ${ }^{1}$ Of these 17 (37\%) who were suitable for radiological intervention, two had tumour-related BCS and one underwent repeated dilatations of a surgically placed mesocaval shunt. Proper patient selection is important, the decision to proceed with radiological intervention being made following assessment of hepatic biopsy, ultrasound and venography findings. Patients unsuitable for venoplasty are those with (i) portal vein thrombosis, (ii) established hepatic fibrosis, (iii) a "spider's web" appearance alone on venography without stenoses of the main hepatic veins, and (iv) complete thrombosis of all hepatic veins. Thrombolysis isineffective in thelast group as thrombosis typically extends out into the fifth order hepatic vein radicals and these smaller veins are difficult to clear effectively due to hepatofugal venous flow. Therefore, if the main veins are cleared, there is usually insufficient inflow to maintain patency. Patients in groups (iii) and (iv) may be suitable forTIPS.

\section{Standard radiological interventional techniques}

Patient preparation includes drainage of ascites, correction of clotting factors and omission of anticoagulant therapy (prior to percutaneous transhepatic procedures). Intravenous antibiotics are routinely administered. Procedures are tolerated well using intravenous sedation/analgesia with appropriate monitoring. The standard radiological techniques are hepatic and IVC venoplasty and/or recanalisation usually performed through a jugular and femoral approach respectively (Figures3a-d). For hepatic vein dilatations, we generally use a 6 F right internal jugular sheath, a general purpose angled catheter to cannulate and a standard angioplasty catheter to dilate the vein (to $12 \mathrm{~mm}$ ) until waisting is abolished. The usual stricture encountered is at the venous ostium, is short, hard to dilate and recoils following deflation of the balloon (Figures 2a,b). Nevertheless, although there may not be much visible widening of the stricture following repeated dilatations (Figure $2 \mathrm{~b}$ ), a reduced pressure gradient, increased blood flow and symptomatic improvement does follow. For IVCobstructions, either one large balloon or up to four smaller balloons placed side to side (introduced via the femoral veins) can be used (Figures 3c,d). Thrombus, if present, in either the IVC or the hepatic veins should be cleared by thrombolysis to dilatation to

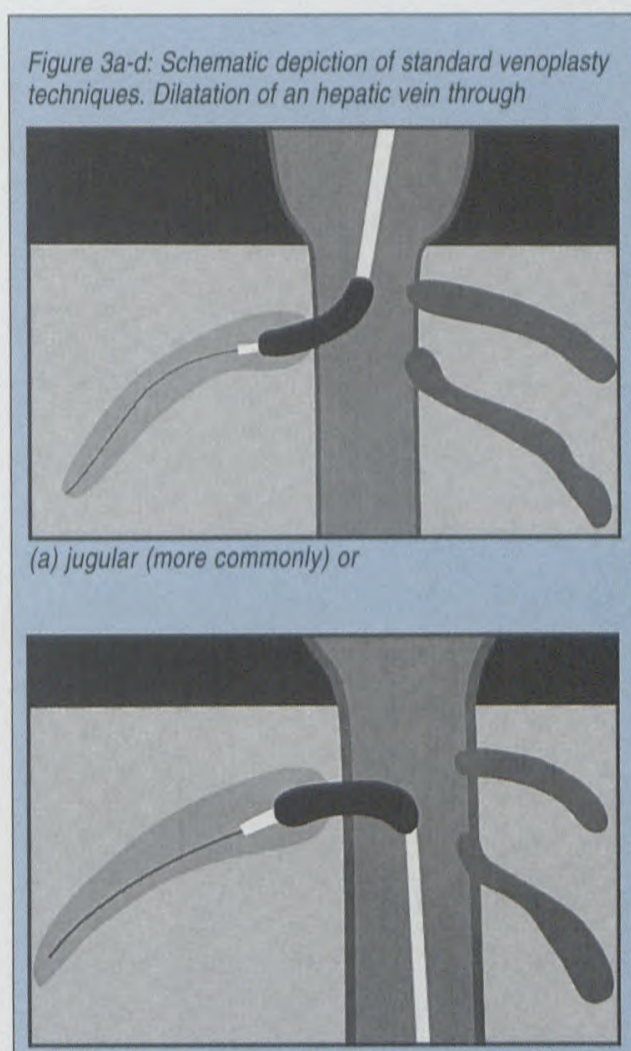

(b) femoral approach. Dilatation of the IVC with

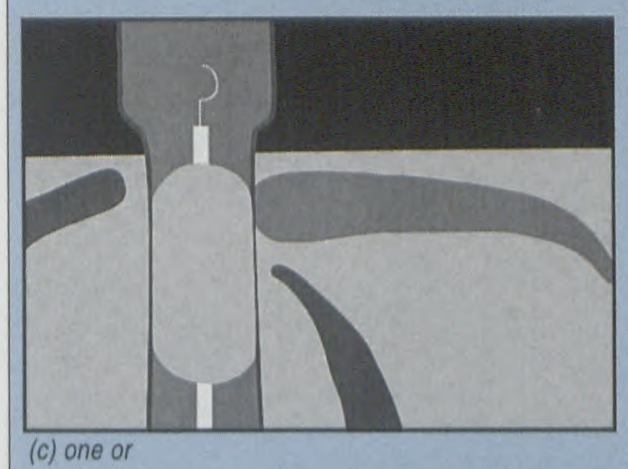

(c) one or

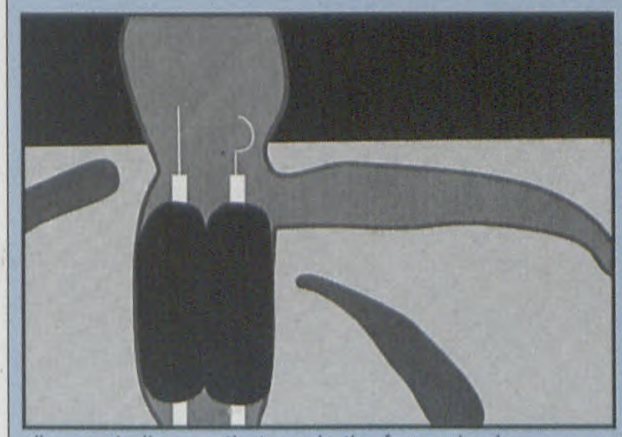

d) more balloon catheters via the femoral vein. 
reduce the risk of thromboembolism. All patients are maintained on long-term anticoagulation though the potential benefits of this are only anecdotal.
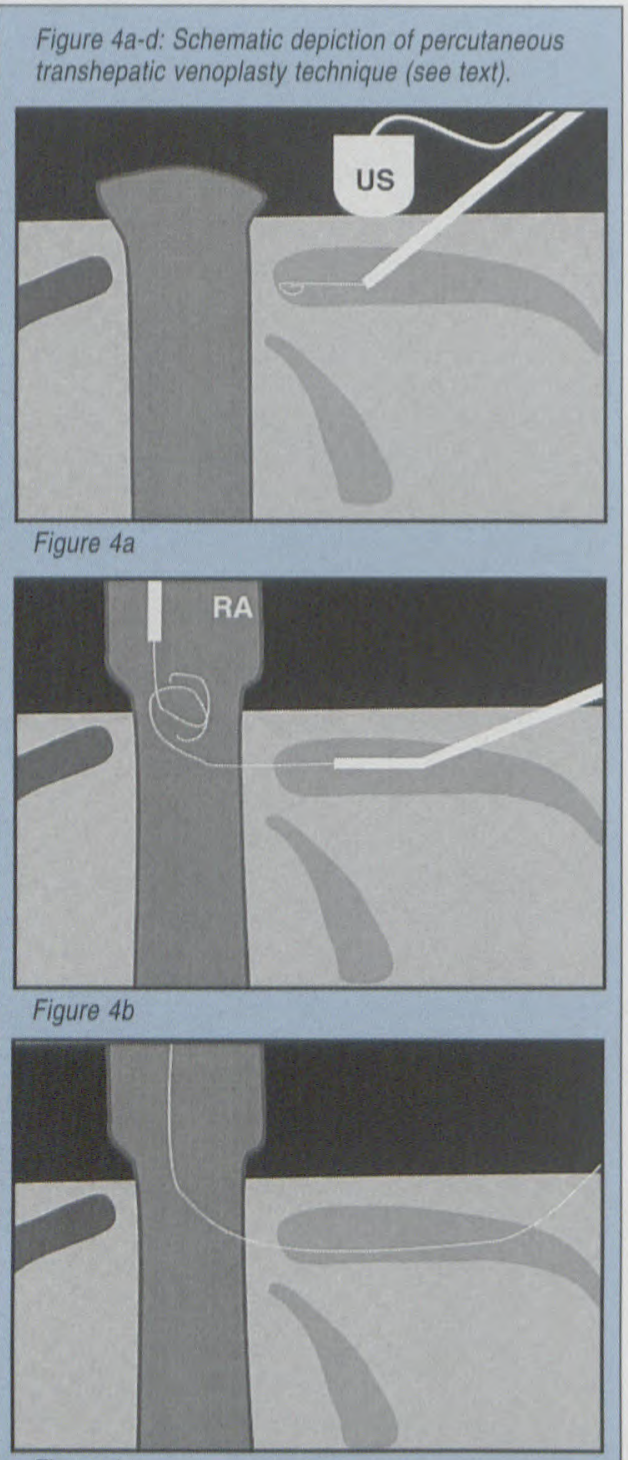

Figure $4 c$

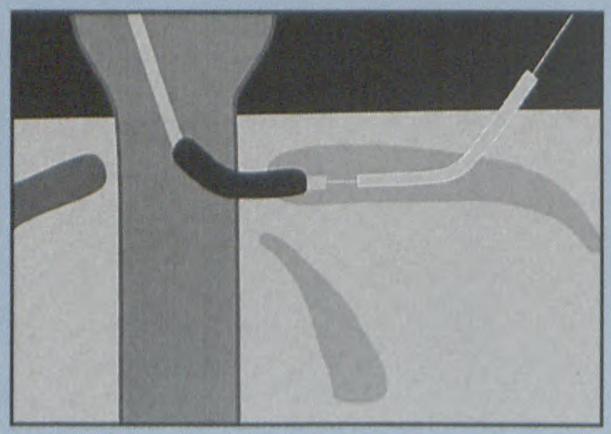

Figure $4 d$

\section{Dealing with completely occluded hepatic ostia}

When the main hepatic venous ostia are occluded a transhepatic approach may be used (Figures 4a-d and 5a,b). Ultrasound allows identification of a suitable patent peripheral hepatic vein which is cannulated percutaneously (Figures 4a,5a). Once the obstructed segment is crossed with a guide wire, the wire can be snared within the RA or IVC and brought out through a jugular sheath (Figures $4 b, c$ ). Anchoring both ends of the wire allows dilatation of the recanalised segment (and, if necessary, stent placement) via the jugularvein (Figures 4d, 5b). ${ }^{39}$ This combined approach leads to an improved recanalisation success rate and reduced the need for large transhepatic tracks thus lowering the risk of bleeding and intrinsic liver damage. It also allows one to steer, under biplane fluoroscopic screening, to a catheter (or inflated balloon) placed in the IVC or HV when attempting a recanalisation from the HV or IVC respectively. A similar technique can be used for a combined femoraljugular vein approach (Figures 6a-d).

\section{Recurrence of hepatic stenoses}

Restenosis is the rule rather than the exception. ${ }^{21,26}$ Five out of $18(28 \%)$ initial angiographic dilatations in our series failed to provide adequate venous return in the first instance due to a combination of restenosis and thrombosis. ${ }^{1}$ Adopting an aggressive attitude to re-intervention during the early treatment period with re-dilatation, use of thrombolytic agents and stent deployment as necessary improved our initial success rate from $72 \%$ to $83 \%$.

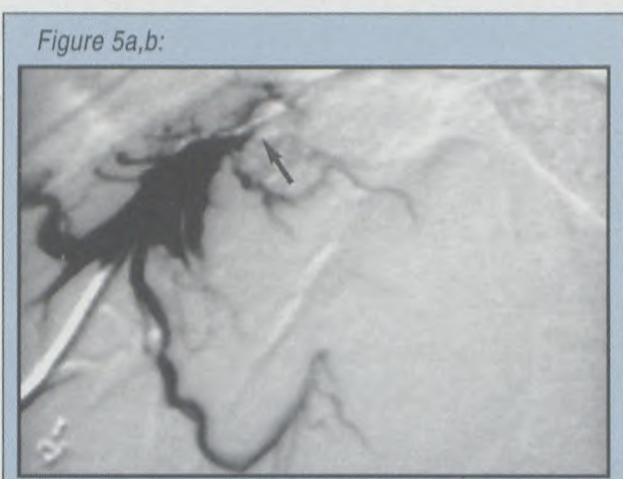

(a) Percutaneous transhepatic venography. There is complete occlusion of the distal middle hepatic vein (straight arrow). Note coil in track of previous attempted cannulation (curved arrow).

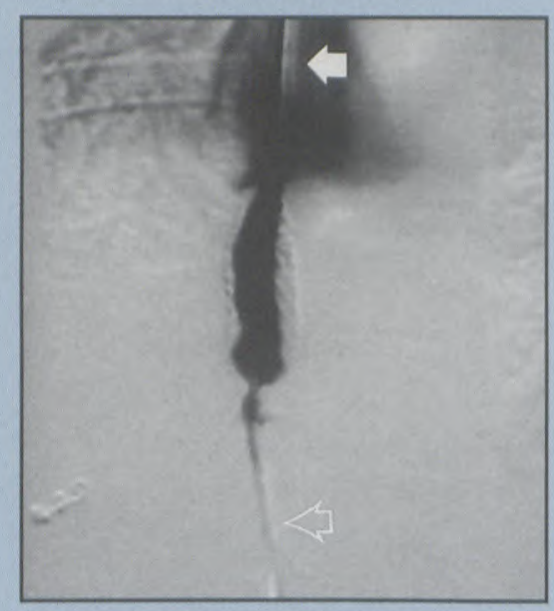

(b) Deployment of stent via the internal jugular vein. Jugular catheter in middle hepatic vein ostium (closed arrow). Transhepatic catheter (open arrow).

Regular review is mandatory. HV restenosis occurred in all patients followed up for longer than 10 months although the duration between restenoses may vary. Generally, IVC stenoses recur less frequently than hepatic vein stenoses, ${ }^{5,21,24}$ The aim of post-intervention surveillance is to re-intervene before critical stenosis or occlusions recur, particularly as they may pre-date clinical or biochemical deterioration. We adopt a policy of regular clini$\mathrm{cal}$ and ultrasound assessment (varying from three-monthly, six-monthly and yearly once the patient is stabilised) supplemented by hepatic venography annually (or sooner should climical, biochemical or ultrasonic deterioration occur). Whilst this policy is uniformly well 


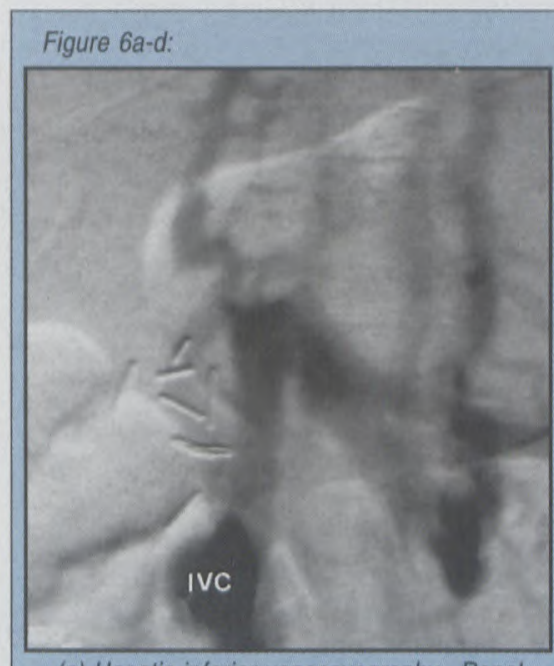

(a) Hepatic inferior venacavography. Renal tumour recurrence in the intrahepatic IVC with diversion into collaterals. It was not possible to enter an hepatic vein from above.

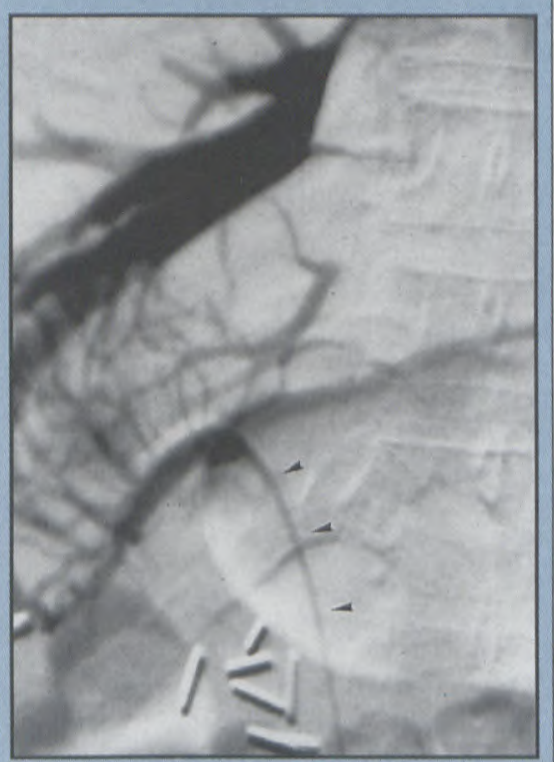

(b) An inferior venous collateral was cannulated (arrowheads).

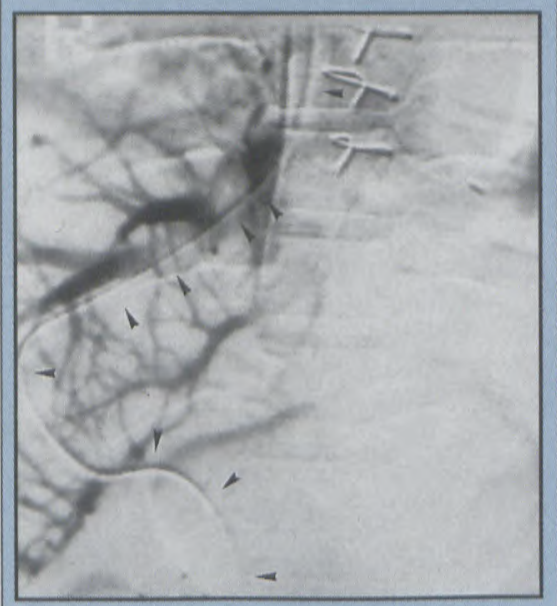

c) A guide wire was passed to the right atrium and snared.

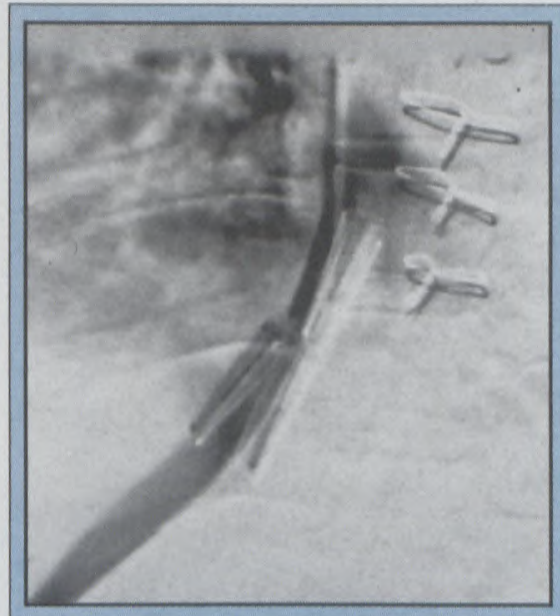

(d) The ostial obstruction was stented via the jugular vein. This patient who presented with severe hepatic encephalopathy remained well for a further 18 months.

tolerated, in occasional circumstances where frequent repeat dilatation is necessary, stent insertion has been undertaken . Radiological intervention does not address the underlying pathophysiology of hepatic venous outflow obstruction. Thus the disease can never be deemed cured and continued review is essential.

\section{Stenting}

IVC stent deployment has been reported more commonly than hepatic vein stenting. We reserve stenting for situations where a) the patient is critically ill and initial dilatations are unsuccessful, b) repeated re-dilatation is required at short intervals on review and c) malignant disease. Where there is significant IVC narrowing, placement of an intrahepatic stent may reduce caval pressure to allow mesocaval shunting rather than more hazardous mesoatrial shunting. As reduction in liver swelling may lead to apparent stent movement, we aim to deploy hepatic vein stents with the tip not protruding much beyond the venous ostium. IVC stents should not extend into the right atrium or the infrahepatic IVC where they will interfere with liver removal (if transplantation is subsequently required).

\section{Safety of radiological intervention}

Very few complications following radiological intervention for BCS have been reported. IVC stent migration is the only significant complication reported to date. ${ }^{19}$ In 49 angiographic dilatations (including 11 recanalisations), we had three serious complications. ${ }^{1}$ In one patient, a small pseudoaneurysm of a right hepatic artery branch presented as haemobilia within days of percutaneous transhepatic intervention. Cephalad migration of two interlaced upper IVC stents into the right atrium by approximately $3 \mathrm{~cm}$ occurred in another patient. This cephalad migration may have precipitated an increased frequency of atrial tachyarrythmias. Myocardial puncture (without haemodynamic compromise) occurred in another patient during recanalisation of an upper IVC occlusion.

\section{Effectiveness of radiological intervention}

Overall, the efficacy of radiological intervention compares favourably with surgical shunting. A "markedimprovement" (i.e. with no symptoms related to hepatic venous outflow obstruction) following radiological intervention wasseen in 10 of the $18(56 \%)$ patients. Some of the patients developed recurrent progressive symptoms in the weeks prior to re-intervention. "Improvement" (i.e. continued mild symptoms of hepatic venous outflow obstruction) was seen in 4 out of $18(22 \%)$ patients again with progressive symptoms prior to re-intervention.

\section{Failure of radiological intervention}

As most reports consist of one or two patients, there is little mention of failed radiological intervention in the literature. 
Radiological intervention failed in 5 out of 18 of our patients, principally during the earlier years of the study. Three of these patients failed early: recurrent thrombolysis( $\mathrm{n}=1)$; failure to recanalise IVC occlusion( $\mathrm{n}=1$ ); and failure to recanalise hepatic vein occlusion( $\mathrm{n}=1$ ). Two latefailures occurred (restenosis, $n=2$ ). Of late, better patient selection, use of a combined transhepatic-transjugular route and stenting have lead to an improved success rate. Should radiological intervention fail, patients can still proceed to surgical shunting or transplantation. Moreover, a limited response to radiological intervention in a patient with severe hepatic failure, may make subsequent surgery less hazardous.

\section{TIPS}

When the main hepatic veins are completely occluded TIPS have been successfully performed (usually through an occluded RHV sinus) as an alternative to surgical portosystemic shunting ${ }^{34-37}$ to relieve portal hypertension and decrease hepatic congestion. Whilst contraindicated in the presence of a concomitant severe upper IVCstenosis, TIPS, as opposed to mesocaval shunting, can be considered when severe caudate lobe hypertrophy exists and does not interfere with subsequent liver transplantation. Asvenous drainage through the hepatic veins is not restored, TIPS carry an inherent risk of portal-systemic encephalopathy although the incidence of this, following surgical portosystemic shunting for Budd-Chiari, is low. ${ }^{39}$

\section{References}

1. Griffith JF, MahmoudAEA, Cooper Set al. Radiological intervention in Budd-Chiari Syndrome: techniques and outcome in 18 patients. Clinical Radiology (in press).

2. Dilawari JB, Bambery P,Chawla Y et al. Hepatic outflow obstruction (Budd-Chiari syndrome). Experience with 177 patients and a review of the literature. Medicine 1994;73:21-36.

3. Klein AS, Sitzmann JV, Coleman J et al. Current management of the Budd-Chiari syndrome. Annals Sungery 1990;212:144-149.
4. Boughton BJ. Hepatic and portal vein thrombosis. Closely associated with chronic myeloproliferative disorders. British Medical Joumal 1991;302:192-193.

5. Hobbs KE. Budd-Chiari syndrome, Lancet 1992:339:115-116.

6. Yang XL, Chen $\mathrm{CR}$, Cheng TO. Nonoperative treatment of membranous obstruction of the inferior vena cava by percutaneous balloon transluminal angioplasty. American Heart Joumal 1992;124:405-412.

7. Hirooka M, Kimura C. Obstruction of the hepatic inferior vena cava. Archives Surgery 1970;100:656-663.

8. Imoka S, Okamato N, Kobayashi et al. Treatment of inferior vena cava obstruction producing Budd-Chiari syndrome. Archives Surgery 1984;119:965-968.

9. Nakamura T, Nakurama S, Aikawa T et al. Obstruction of the inferior vena cava in the hepatic portion and the hepatic veins. Angiology 1968;19:478-498.

10. Kage M, Arakawa M, Kojiro Met al. Histopathology of membranous obstruction of the inferior vena cava in the Budd-Chiari syndrome. Gastroenterology 1992;102:2081-2090.

11. Tavill AS, Wood EJ, Kreel L et al. The Budd-Chiari syndrome: correlation between hepatic scintigraphy and the clinical, radiological, and pathological findings in nineteen cases of hepatic venous outflow obstruction. Gastroenterology 1975;68:509-518

12. Grant EG, Perrella R, Tessler FN et al. Budd-Chiari syndrome: the results of duplex and color Doppler imaging. American Joumal of Roentgenology 1989;152:377381

13. Hosoki T, Kuroda C, Tokunaga Ket al. Hepatic venous outflow obstruction: evaluation with pulsed duplex sonography. Radiology 1989;170:733-737.

14. Kane R, Eustace S. Diagnosis of Budd-Chiari Syndrome: comparison between sonography and MR angiography. Radiology 1995;117-121.

15. Yamada R, Sato M, Kawabata Met al. Segmental obstruction of the hepatic inferior vena cava treated by transluminal angioplasty. Radiology 1983;149:91-96.

16. Parker RGF. Occlusion of the hepatic veins in man. Medicine 1959;38:369-402.

17. Eguchi S, Takechi Y,Asano K. Successful balloon membranotomy for obstruction of the inferior vena cava. Surgery 1974;76:837-840.

18. Chan P, Lee CP, LeeYH. Complete membranous obstruction of the inferior vena cava treated successfully by King's bioptome and balloon angioplasty. American Joumal of Cardiology 1993;72:241-243.

19. Furui A, Sawada S, Toshiyuki I et al. Hepatic inferior vena cava obstruction: treatment of two types with Gianturco expandable metallic stents. Radiology 1990;176:665-670

20. Meier III WL, Waller III RM, Sones Jr PJ. BuddChiari web treated by percutaneous transluminal angioplasty. American Journal of Roentgenology 1981;137:1257-1258

21. Nishikawa M, Seki K, Miyoshi S et al. Treatment of the Budd-Chiari syndrome in polycythemia ruba vera by repeated percutaneous transluminal angioplasty of a hepatic vein stenosis. Postgraduate Medical Journal 1982:58:511-514.

22. Jeans WD, Bourne JT, ReadAE. Treatment of hepatic vein and inferior vena caval obstruction by balloon dilatation. British Joumal of Radiology 1983;56:687-689.
23. Greenwood LH, Yzirarry JM, Hallett JW, Scoville GS. Urokinase treatment of Budd-Chiari syndrome. American Joumal of Roentgenology 1983;141:1057-1059.

24. Uflacker R, Francisconi CF, Rodriguez MP, Amaral NM. Percutaneous transluminal angioplasty of the hepatic veins for treatment of Budd-Chiari syndrome Radiology 1984;153:641-642.

25. Sholar PW, Bell WR. Thrombolytic therapy for inferior vena cava thrombosis in paroxysmal nocturnal haemoglobinuria. Annals of Internal Medicine 1985;103:539-541.

26. Sparana J, Chang J, Trasi S, Bonanno C. Treatment of the Budd-Chiari syndrome with percutaneous transluminal angioplasty. Case report and review of the literature. American Joumal of Medicine 1987;82:821-828.

27. Furui S, YamauchiT, Ohtomo Ketal. Hepatic inferior vena cava obstructions: clinical results of treatment with percutaneous transluminal laser-assisted angioplasty. Radiology 1988;16:673-677.

28. Vickers CR, West RJ, Hubscher SG, Elias E. Hepatic vein webs and resistant ascites. Diagnoses, management and implications. Journal of Hepatology 1989;8:287-293.

29. Lois JF, Hartzman S, McGlade CT et al. BuddChiari syndrome: treatment with percutaneous transhepatic recanalisation and dilatation. Radiology 1989;170:791-793.

30. Feigin DR, Glickson M, Varstending A et al. Familial Budd-Chiari syndrome due to membranous obstruction of the right hepatic vein treated with transluminal angioplasty. American Journal of Gastroenterology 1990;85:94-97.

31. Wakao F, Takayasu K, Muramatsu Y, Nawano S. MR evaluation of Budd-Chiari syndrome treated by percutaneous transluminal angioplasty (letter). American Joumal of Roentgenology 1990;154:1351.

32. Weernink EEM, Huisman AB, Ten Napel CHH Treatment of Budd-Chiari syndrome by insertion of wall-stent in hepatic vein (letter). Lancet 1991;338:644

33. Lopez Jr RR, Benner KG, Hall Let al. Expandable venous stents for treatment of Budd-Chiari syndrome Gastroenterology 1991;100:1435-1441.

34. Ochs A, Sellinger M, Haag K et al. Transjugular intrahepatic portosystemic stent-shunt (TIPS) in the treatment of Budd-Chiari syndrome. Journal of Hepatology 1993;18:217-225.

35. Peltzer MY, RingEJ,LaBerge JMet al. Treatment of Budd-Chiari syndrome with a transjugular intrahepatic portosystemic shunt. Journal of Vascular Interventional Radiology 1993;4:263-267.

36. Hacking CN, Sanderson AJ, Arthur MJP. BuddChiari syndrome - treatment with transjugular intrahepatic portosystemic shunt (TIPS). Journal of Vascular Interventional Radiology 1994;9:121-124.

37. Fujimoto M, Moriyasu F, Someda H et al. BuddChiari syndrome; recanalization of an occluded hepatic vein with percutaneous angioplasty and a metallic stent. Joumal of Vascular Interventional Radiology 1993;4:257. 261.

38. Martin LG, Henderson JM, Millikan WJ et al. Angioplasty for long-term treatment of patients with Budd-Chiari syndrome. American Journal of Roentgenology 1989;154:1007-1010.

39. Brems J, Hiatt J, Millis J et al. Effect of prior portosystemic shunt on subsequent liver transplantation. Annals Surgery 1989;209:52-56. 